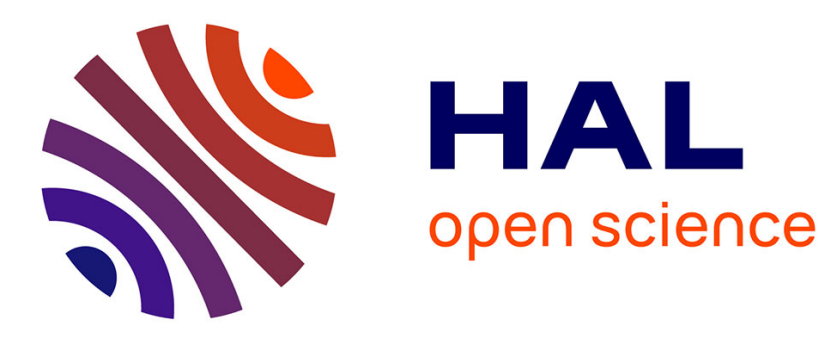

\title{
A Hardware in the Loop module in an IEC61850 Co-Simulation Platform for advanced substation automation system tests
}

Mohamad Haffar, Jean-Marc Thiriet, Mohamad El-Nachar

\section{- To cite this version:}

Mohamad Haffar, Jean-Marc Thiriet, Mohamad El-Nachar. A Hardware in the Loop module in an IEC61850 Co-Simulation Platform for advanced substation automation system tests. EnergyCon 2010 - IEEE International Energy Conference and Exhibition, Dec 2010, Manama, Bahrain. paper 1569328789. hal-00559633

\section{HAL Id: hal-00559633 \\ https://hal.science/hal-00559633}

Submitted on 26 Jan 2011

HAL is a multi-disciplinary open access archive for the deposit and dissemination of scientific research documents, whether they are published or not. The documents may come from teaching and research institutions in France or abroad, or from public or private research centers.
L'archive ouverte pluridisciplinaire HAL, est destinée au dépôt et à la diffusion de documents scientifiques de niveau recherche, publiés ou non, émanant des établissements d'enseignement et de recherche français ou étrangers, des laboratoires publics ou privés. 


\section{A Hardware in the Loop module in an IEC61850 Co- Simulation Platform for advanced substation automation system tests}

\author{
Mohamad Haffar \\ Euro System \\ Varces, France \\ mohamad.haffar@euro-system.fr
}

\author{
Jean-Marc Thiriet \\ GIPSA-lab (UMR 5216), UJF \\ Grenoble, France \\ jean-marc.thiriet@ujf-grenoble.fr
}

\author{
Mohamad Nachar \\ GIPSA-lab (UMR 5216) \\ Grenoble, France \\ mohammad.el-nachar@gipsa- \\ $\underline{\text { lab.grenoble-inp.fr }}$
}

\begin{abstract}
- the testing of architectures is nowadays one of the most a critical issue for Substation Automation System world. Various aspects, related to the IEC61850 requirements, should be tested and checked in order to validate architecture. The first aspect consists in validating the conformity of the IEC61850 implementation inside devices according to the standards requirements. The second aspect consists in validating IEC61850 applications compliance according to the predefined functional specifications. Some devices and parts of the architecture can of course be tested "physically"; but in the design phase or when the actual architecture cannot be checked directly, modeling is helpful. In our research study we propose a co-simulation approach based on several components allowing the realization of substation automation system advanced tests. This paper describes the importance and the design implementation of hardware in the loop component allowing the combination of real and virtual devices.
\end{abstract}

\section{INTRODUCTION}

In the early 1990 communication technologies and protocols began to appear in Substation Automation System (SAS) application. In 2004, a new worldwide standard of communication IEC61850 was introduced in the majority of substation automation systems carrying out new innovation prospects. The interoperability between devices from different manufacturers as well as the insurance of the overall security of SAS architecture through its communication network becomes now attainable with this new communication standard. In addition, object data modeling is used inside IEC61850 equipments to replace the aspect of non significant addresses. The standard provides a stack of services in order to obtain a self description of the internal objects and communication capabilities of the device; this point permits to reduce the time of developing supervision control and data acquisition applications by facilitating the identification of the device capabilities.

IEC61850 authorizes the distribution of functionalities between devices. This aspect permits the exchange of real time inter-equipment messages over the communication network to provide advanced services for electrical substation process (e.g. interlocking, load shedding, auto transfer Switch, etc...).

Unlike previous communications protocols, distribution functionality requires a configuration of the device's dataflow which was not possible previously. Standard XML configuration files are published with each constructor's device to allow the configuration of its communications capabilities and parameters setting. In addition to the device configuration file, a global configuration file for the overall SAS network is also provided by the IEC 61850 standard; this file is called SCD (i.e. Substation Configuration Description file). The generation of this SCD file in a multiproducts/constructors architecture implies the need of an architecture designer. This new aspect of configuration relies on the network capacity and the devices capabilities connected to this network; thus it must be studied and tested before its implementation.

Being the unique standard used in the SAS network, devices from different constructors will be able to dialog in order to exchange information and services. This feature offers to the engineering staff more liberty in choosing their Intelligent Electronic Devices (IED) (e.g. protection relay, power meter ...). However, the heterogeneity of IEDs inside SAS architecture yields complexities to the Factory Acceptance Testing (FAT) which becomes difficult to realize due to the dispersed location of the switchboards suppliers. An exhaustive FAT must be provided with each project development in order to minimize time of site commissioning. This point is an essential key demanded by the end users.

End Users must have a realistic view about the demanded SAS architecture tests according to their desired requirements. Thus they should comprehend the limitation scope of the conformance tests provided by the international certification organizations such as Kema [2] versus a complete successful 
implementation of IEC 61850 SAS architecture which should be coordinated by all involving parties.

\section{LIMITATIONS SCOPE OF STANDARD CERTIFICATION TESTS}

A generic testing plan for an IEC61850 SAS architecture shall include four testing categories: the conformance testing of a device, the distributed functionalities testing, the interoperability testing and the global system performance evaluation [1]. Witness and hold points shall be specified in the testing plan for carrying out inspections that verify the quality of tests.

Utility Communications Architecture International Users Group (UCAIUG) has established a conformance testing program for IEDs which permits the certification of the internal object data modeling, communication oriented services and the global configuration online testing.

These conformance tests could be considered as parts of the device conformance testing. Therefore distributed functionalities, interoperability and global system performance evaluation tests are still left unanswered. Guidelines are given by the international certification organization to accomplish these advanced tests.

New tools and advanced models should be provided to fill the gap of tests. These tools should also permits the cohabitation of real devices of a switchboard supplier with device modeling of the missing switchboard to ensure an advanced FAT in a heterogeneous SAS architecture.

Based on the guidelines of advanced tests proposed by international certification organization, our work deals with a Co-Simulation Platform to accomplish a complete IEC 61850 advanced test. This platform is constructed with models of any IED constructor. Real communication IEC61850 services and internal data objects are implemented inside each IED model. Several components such as fault detection and fault rectification application, standard model software configuration, external electrical process simulation acting as software in the loop [7] and Hardware in the Loop (HITL) module will be implemented in this Co-Simulation platform.

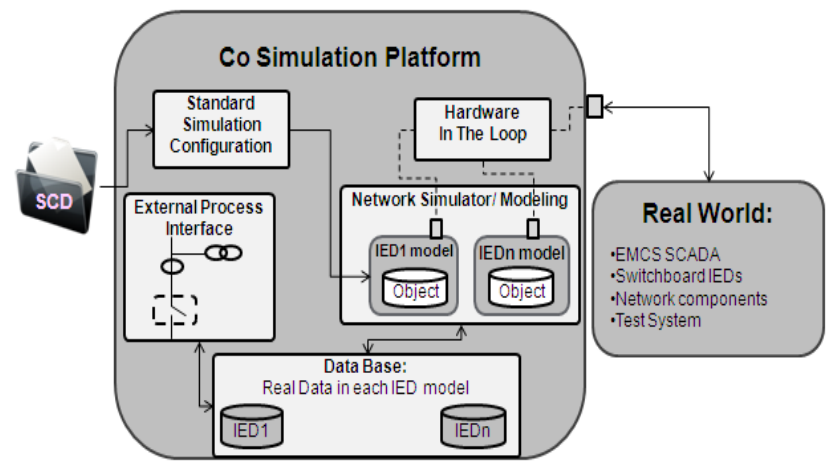

Figure 1. Co-Simulation platform architecture

Fault Detection and Fault Rectification (FDFR) application is used for the validation of structural statements of all standard configuration files in the SAS architecture. More particularly, IED Capabilities Description (ICD) and
Configuration IED Description (CID) files will be tested in offline mode according to the specification of the standard's $7^{\text {th }}$ part.

The major purpose of the Standard Model Software Configuration (SMSC) is to generate and configure any IED model coming from any supplier by importing its configuration file. This provides the interoperability of the Co Simulation Platform at the modeling stage.

External Electrical Process Simulation (EEPS) is a Software In The Loop 'SITL' component that sends Inputs/ Outputs process value (e.g. voltages, currents, load, status trip, default generation) inside the simulation platform to test the IED model behavior. This component aims to ensure global configuration testing for each configured model device inside the Co Simulation Platform.

The final component HITL is the main subject of this paper. We will explain in detail, the importance of including HITL as a component in the Co-Simulation platform, the development and implementation detail and finally the test of the HITL module in an IEC 61850 application.

\section{NETWORK SimULATION SOFTWARE}

Modeling and simulating a networked system requires a network simulator that takes into account all major contributions including protocols, medium access control technology, network load, communication imperfections, timeouts and packet losses etc. Popular network simulators include NS2, OMNET, Qualnet, OPNET etc. OPNET Modeler is chosen in our research because it implements a large number of libraries of standard equipment (e.g. switches and link models) as well as proprietary models, which is useful for simulation and reduces the development time [10]. Object oriented modeling approach is used in OPNET in order to facilitate the construction of models.

A network device is modeled as a node which is composed of many modules connected by packet streams or static wires. Each module aims to represent specific aspect of the node's behaviour such as data creation, data storage, data processing or routing (IP layer), data transmission (Transport layer), etc. Thus the construction of each layer of the TCP IP stack is made in OPNET modules.

The process is programmed using the finite state machine (FSM) technology. This approach facilitates the supporting of the implementation of applications, algorithms and queuing policies. States and transitions graphically define the progression of a process in response to events. Each state in process model contains embedded $\mathrm{C} / \mathrm{C}++$ code, supported by an extensive library of functions designed for network programming [9].

\section{HARDWARE IN THE LOOP}

Hardware in the Loop is a simulation component that permits real network devices to communicate with simulated network devices by making the appropriate packets conversion. This component can support more than one physical interface in a simulation machine which allows 
mapping of different network addresses in the simulated network.

According to the placement of the simulation machine, three basic scenarios can be provided using this module: the real to real, simulated to simulated and simulated to real. In real to real scenario the simulation is inserted between two real networks, in simulated to simulated scenario a real network connects two simulation worlds and finally in simulated to real scenario a simulation components inside the simulation machine are directly connected to a real network components.

\section{A. Platform construction phase}

Each IEC61850 server (i.e. device or model) is delivered with its Model Implementation Conformance Statement 'MICS' and Protocol Implementation Conformance Statement 'PICS'. MICS documentation describes the capabilities of the device in term of functionalities described in object format. However PICS is another documentation which describes the IEC61850 services supported by the device or its model. The conformance tests of a device is based on tests that cover the conformity of object data modeling as well as the conformity IEC 61850 Abstract Communication Services Interfaces 'ACSI' according to PICS and MICS documentations. Our Co-Simulation platform is based on modeling of device communications. The increase of confidence in our platform is an important point in our research study. To accomplish this point, compliance tests should be provided to IEDs models according to the IEC 61850 flowchart tests.

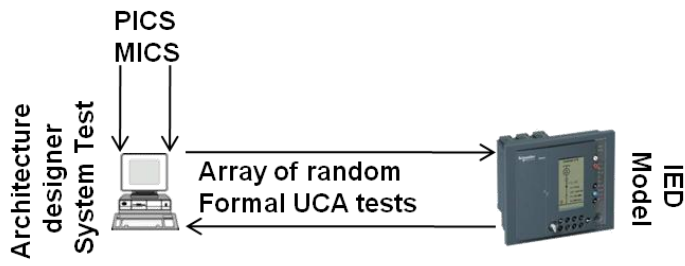

Figure 2. Compliance tests for an IEC61850 server

HITL simulated to real scenario is used in this case to provide the connecting of IED model included in the CoSimulation platform to an external test system that sends an array of tests to the model to verify the two types of conformance. Being external to the simulation world, packets sent by the test system should be converted from real to simulated and reach the IED model. At its turn, the IED model should respond to the tests packets. Simulated to real scenario intervenes to ensure conversion of packets from real to simulation and vice versa. At this stage an HITL component permits the increase of confidence in the modeling strategy.

\section{B. Project study phase}

During the SAS project study phase, the architecture platform will provide a solution for defining SAS network topology and coordinating configuration files to achieve end user's specifications and increase SAS reliability [6]. Therefore, the architecture designer will be able to modify the SCD configuration file of the overall architecture in order to reach the system required performance.
HITL real to real scenario is used inside the platform to interface a simulated network between two real worlds. Using this scenario, punctual studies about the reliability of inter exchanging real time message between networks devices will be given. Multi protection devices will be interconnected via a simulated network using HITL Real-to-Real scenario. Therefore, LAN connecting the IEDs will be simulated to produce three types of network delays (congestion, latency and bandwidth). This is done by generating unused traffic load in the simulated network and changing networks elements and architecture. This leads to the evaluation of the End To End time performance of real time messages from the time the publisher IED sends its real time control message until the time of receiving the message on the subscriber IED side.

\section{Factory acceptance test phase}

The Factory Acceptance Test 'FAT' is an essential phase in each project development. During FAT period, application compliance will be validated according to the functional specification defined in the period of project study. The global system performance is also tested during this phase.

IEC 61850 leads for a new approach of FAT. Data flows exchanged between IEDs is a new type of communication provided by the IEC61850. This type of communication spawns two kinds of additional tests, horizontal and network capabilities tests that will be added to the classical FAT tests (when 61850 is not used). The origin of this type of communication is the distribution of functionalities provided by the IEC61850.

Distributed functionality is based on Publisher/Subscriber communication and permits to solve peer to peer power management system application (e.g. load shedding, bus blocking, etc...) through the communication network. This leads to have three important points to test during FAT:

- Configuration tests of the Publisher

- Configuration tests of the Subscriber

- Network capabilities tests to ensure that End To End of peer to peer messages correspond to critical distributed application delays.

However, as IEC 61850 permits interoperability between devices, it should be noticed that during FAT period some switchboard devices could be missed because of the constructor's heterogeneity. Another aspect to take into consideration is that network components and architecture are present during Site commissioning and generally not during FAT period.

HITL component intervenes for connecting IED models to real IED through a simulated network and making advanced FAT realizable. In FAT case, two scenarios must be combined for the realization of exhaustive FAT: the Real To Real and the simulated to real as shown in the Fig. 3. 


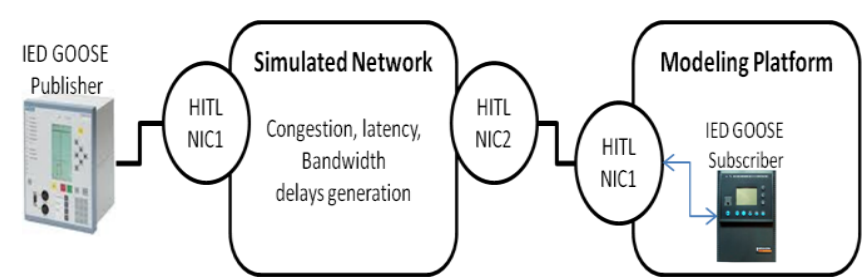

Figure 3. Exhaustive IEC61850 FAT using HITL

\section{IMPLEMENTATION OF HARDWARE IN THE LOOP}

HITL node is based on an edge Router interface which is used inside the simulation platform in order to provide the workflow for exchanging packets between simulated and real devices. The detailed of this workflow is shown in the Fig. 4.

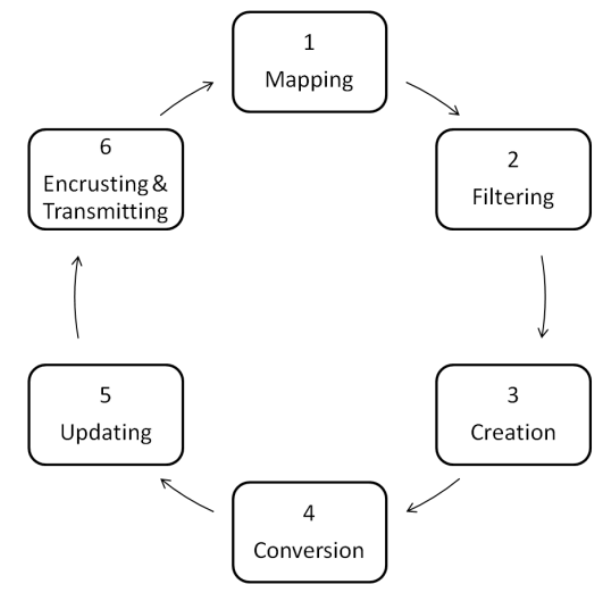

Figure 4. HITL workflow cycle

The first important job of the edge interface router is the mapping of external network interface of the simulation machine to the network interface of the appropriate device model. HITL allows multiple interfaces to map different network addresses in the simulated network.

HITL module has many associated attributes that permits to describe the HITL behavior. One of these attribute is used for filtering packets received by HITL in order to reject unexpected packets and allocate memory for expected real world packets. If the packet is accepted, the edge router enter the creation phase where it constructs the skeleton of the simulated or real packet weather if the packet received is real or simulated.

To succeed the phase of copying the contents of packet fields between the two formats, HITL requires a predefined well known protocol format includes in the Standard Model Library packets. Otherwise, if the packet received is not a part of the library a custom code must be written for providing the conversion of the unsupported protocol format packets else it will be dropped by the edge router and will not enter the simulation world. The protocols format packets supported or partially supported by HITL are the ones associated to standard TCP/IP layers as shown in figure 5.
As described in the introduction, the Simulation Platform is used for solving Power Management System 'PMS' issues, thus HITL must provide conversion of protocols associated to PMS more particularly the IEC 61850 standard. IEC 61850 packets format and other industrial protocols are not included inside the Standard Model Library Packets thus the conversion of application layer packets are not provided with a nominal HITL.

Our customized user code is developed to provide the conversion of protocol application packets. In addition, the code is also used to fill in uncompleted conversion points of transport packets by updating (i.e. phase 5 of the HITL workflow shown in figure 4) TCP missing fields which are associated to the application (e.g. data length field).
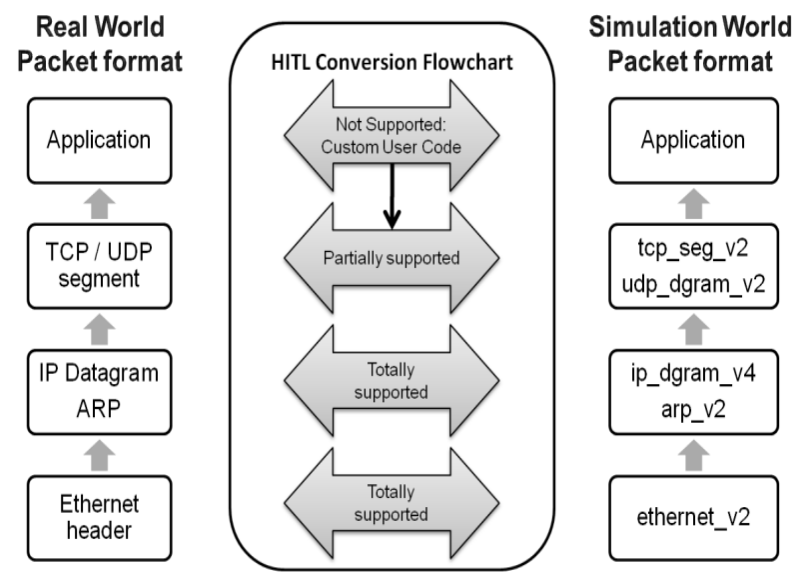

Figure 5. HITL conversion supported packets

A surplus point of our custom code is that it has been developed in a way to make use of HITL with any industrial hardware device taking into consideration that it is possible to have heterogeneous power management architecture combining the IEC 61850 and other industrial protocols such as Modbus or others.

After a successful conversion and updating of partially supported packets format, the HITL encrusts real or simulated packet into a SITL conversion block and routes it finally to the appropriate model if the original packet is real or to the external world if the original packet is simulated.

\section{MidDLEWARE MODULE FOR TCP CONNECTIONS SIMPLIFICATION}

Any IEC 61850 device must be modeled to support a client/server over the TCP/IP stack. OPNET Modeler disposed two process models tcp_manager_v3 and its child tcp_conn_v3 in order to establish TCP network connections. To construct any device model, modeler developer must construct the application module corresponding to the application device. To exchange messages and services with other device models inside the Co-Simulation platform or with external real devices using HITL, application module must established active and/or passive connections weather if the device is a client and/or a server. The procedure of managing connections is based on sending commands to the tcp_manager_v3 OPNET process and receiving indications 
corresponding to the request sent. This is done by integrating two externals functions library inside the process of the application and using their appropriate functions. This procedure should be done for each network models and Process application should be modified each time the connections parameters changed. This reason had leaded us to construct a connection middleware module to manage the model connections. The essential objective of this middleware is to eliminate the complexity of managing network connections by replacing the process programming with a simple object oriented configuration.

This way of configuration is based on compound attribute associated to the module which aims to define configurable parameters settings to be used inside the middleware process module. This notion is used to configure the number of passive and active connection and the parameters of each connection (IP address, port number, etc...). Once configured, the middleware module uses external functions libraries to establish TCP or UDP connections weather with real device or a simulated device inside the Co-Simulation Platform. At the end, the middleware connection module sends to the upper layer (i.e. application) the connection ID to use it and send packets it through the middleware.

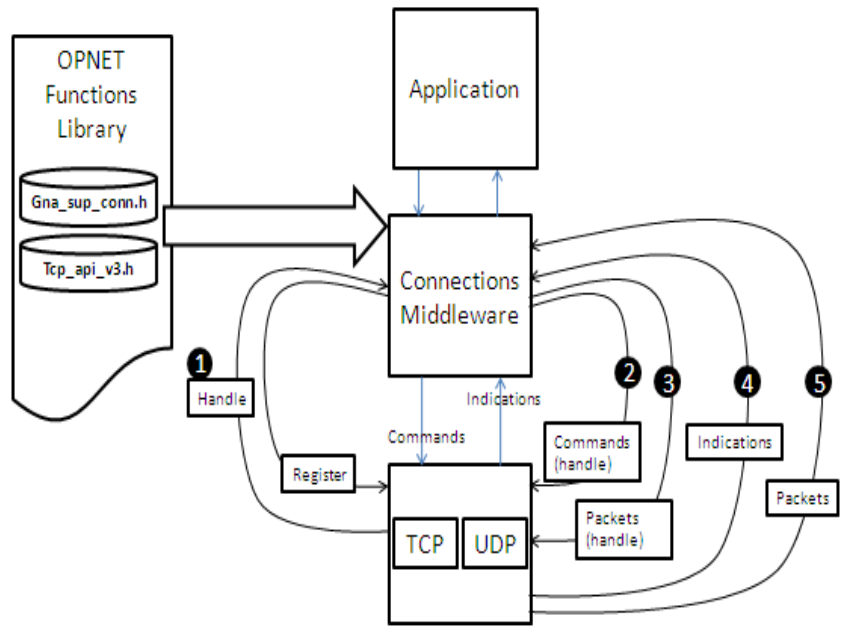

Figure 6. Connection middleware layer

\section{SimULATION TEST}

The simulation test described in this paragraph shows how HITL module is used in order to test the compliance of data modeling inside an IED model.

\section{A. IEC61850 modeling concept}

The IEC61850 includes a comprehensive set of data models for substation (and beyond) functionality. The models are, in general, constructed from a set of common data classes (defined in part 7-3 of the standard). The common data classes are used to build data objects, which are then clustered in logical node classes. Each logical node class represents some basic application function. Instances of the logical node classes are in turn combined to construct logical devices. A physical network device may contain an arbitrary number of logical devices. The set of classes (common data classes, data objects, and logical node classes) define the network visible data types (network interface), along with some standardized behavior, definitions, and configuration parameters [4]. One of the most important points in testing a physical or model device is to verify the compliance of its data object modeling. The paragraph below describes how HITL module is used for accomplishing this point.

\section{B. HITL for testing object modeling}

When a physical or a model IED is constructed, it is delivered with its PICS and MICS documentation which describe the capability of the IED in term of functionalities and services. Before delivering the IED it is crucial to verify the conformity of these two documentations according to the IEC61850 specification to increase confidence in implementation (i.e. interoperability). Verifying the MICS of the IED consists to check:

- The existence of the functionalities defined in the MICS

- The compliance of the object modeling according to IEC 61850 standard dictionary (i.e. part 7.3 and 7.4)

The testing of IEC 61850 implementations is potentially a large task, given the large scope of the standard services and data models. IEDScout is a universal IEC 61850 test systems [8] that can be connected to any IEC 61850 device (server) and provides many useful functions for verifying data model, reading and writing data, reporting, along with GOOSE/GSSE publishing and subscribing.

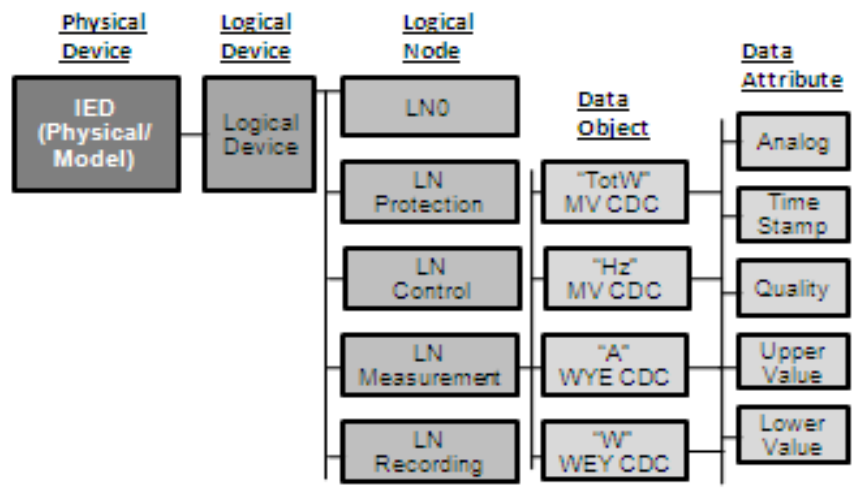

Figure 7. Simple server MICS representation

A simple IEC 61850 measurement unit functionality has been implemented in our IED model to test the data object implementation. The model IED does not implement the functionality, but it is important to note that even unmapped model elements are implemented in the IEC61850 side of the model. The MMXU logical node contains four standard measurement unit data objects: TotW, A, W, Hz which permits to calculate the energy, current, power and frequency. Fig. 7 shows the representation of MICS IED model. 


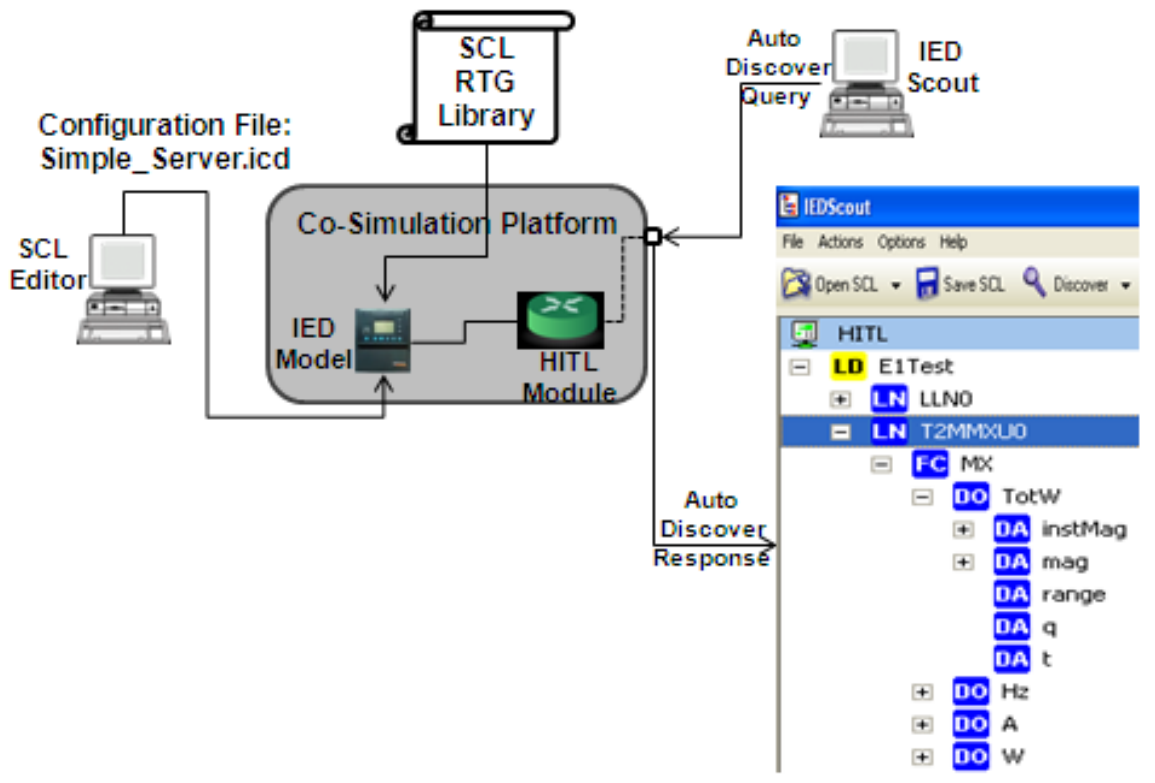

Figure 8. Object implementation testing platform

In Fig. 8 it is shown that the generation of IEC61850 dictionary relies on the implementation of essential points:

- An ICD configuration file which describes the content of the server (i.e. simple server in our example). This file is generated by the SCL standard editor that produces IEC61850 configuration files.

- SCL RTG Library which includes predefined IEC 61850 code that aims to generate on run time the IEC61850 object dictionary according to the ICD configuration file and to implement the auto discover functionality.

For testing the conformity of implementing the server data model, HITL is used to connect the real IEDScout test system to the IED model using real to simulated scenario. The aim idea consists on showing the content of the IED model and identifying the object included in the model that are conformed or not to the standard dictionary. The test system sends an auto discover query to the configured IED model in order to discover the content of its standardized data object. This query passes through the HITL component as shown in Fig. 8. At its turn, the IED model sends back a file transfer response containing its entire data object content, the response reaches the real world also through the HITL component. In our example, the test system shows clearly the content of the simple server included in Fig. 7. This allows us to certify the implementation of simple server data object.

\section{CONCLUSION}

Because of the complexity of the new IEC61850 standard, a flowchart testing plan must be clearly defined based on advanced tools. The main aim of our research is to achieve the entire Platform in order to provide a punctual study for the SAS global performance and to ensure advanced tests in order to increase confidence before any site commissioning phase. This paper was mainly dealt with implementing hardware in the loop inside the Co-Simulation Platform. The essential work of the HITL component is to unify simulation world and real world to make the platform more realistic.

\section{REFERENCES}

[1] IEC; IEC61850 Communication Networks and Systems in substations, part 10: Conformance Testing (IEC61850-10), 2005; http://www.iec.ch

[2] E. A. Udren, D. Dolezilek, "IEC61850: Role of Conformance Testing in Successful Integration"; KEMMA T\&D and Schweitzer Engineering Laboratories 2006

[3] C. Jiongcong, R.Yanming, G. Xinhua, J. Yangjun, "The research on Conformance Testing Platform of Numerical Substation," Elec. Dist., CICED 2008, Decembre 2008

[4] A.P. Apostolov, "Modeling Systems with Distributed Generators in IEC 61850," Power Sys. Conf, PSC '09. Clemson, SC, March 2009.

[5] F. Qingdong, F. Siyao, "Application of IEC61850 in substation automation system," Elec. Dist., CICED 2008, Decembre 2008.

[6] IEEE C37.115-2003, "IEEE Standard Test Method for Use in the Evaluation of Message Communications Between Intelligent Electronic Devices in an Integrated Substation Protection, Control, and Data Acquisition System," IEEE Power Engineering Society, New York, June 2004

[7] R. Martinez, W. Wu, K. Mcneill, "hardware and software-in-the-loop techniques using the opnet modeling tool for jtrs developmental testing," MILCOM, Monterey, October 2003.

[8] A. Apostolov, B. Vandiver, "Functional Testing of IEC 61850 Based IEDs and Systems," Power System Conference and Exposition, Los Angeles, PES2004, October 2004.

[9] Opnet online documentation, http://www.didierfavre.net/OpnetModeler.htm

[10] J.Guo, W.Xiang, S.Wang; "Reinforce Networking Theory with OPNET Simulation," Journal of Information Technology Education, MI, USA, 2007. 